\title{
Spike-Frequency Adaptation Separates Transient Communication Signals from Background Oscillations
}

\author{
Jan Benda, André Longtin, and Len Maler \\ Department of Cellular and Molecular Medicine, Faculty of Medicine, University of Ottawa, Ottawa, Ontario, K1H 8M5 Canada
}

Spike-frequency adaptation is a prominent feature of many neurons. However, little is known about its computational role in processing behaviorally relevant natural stimuli beyond filtering out slow changes in stimulus intensity. Here, we present a more complex example in which we demonstrate how spike-frequency adaptation plays a key role in separating transient signals from slower oscillatory signals. We recorded in vivo from very rapidly adapting electroreceptor afferents of the weakly electric fish Apteronotus leptorhynchus. The firing-frequency response of electroreceptors to fast communication stimuli ("small chirps") is strongly enhanced compared with the response to slower oscillations ("beats") arising from interactions of same-sex conspecifics. We are able to accurately predict the electroreceptor afferent response to chirps and beats, using a recently proposed general model for spike-frequency adaptation. The parameters of the model are determined for each neuron individually from the responses to step stimuli. We conclude that the dynamics of the rapid spike-frequency adaptation is sufficient to explain the data. Analysis of additional data from step responses demonstrates that spike-frequency adaptation acts subtractively rather than divisively as expected from depressing synapses. Therefore, the adaptation dynamics is linear and creates a high-pass filter with a cutoff frequency of $23 \mathrm{~Hz}$ that separates fast signals from slower changes in input. A similar critical frequency is seen in behavioral data on the probability of a fish emitting chirps as a function of beat frequency. These results demonstrate how spike-frequency adaptation in general can facilitate extraction of signals of different time scales, specifically high-frequency signals embedded in slower oscillations.

Key words: adaptation; high-pass filter; communication; receptor neuron; electrosensory system; weakly electric fish

\section{Introduction}

Many neurons will respond to the onset of a constant input with spike-frequency adaptation (i.e., a gradual reduction of the firing frequency). Neuronal adaptation is believed to underlie the commonly observed behavioral habituation of sensory systems to constant stimulation. There are numerous processes that can produce spike-frequency adaptation, including both intrinsic mechanisms and network interactions such as inhibition or synaptic depression. Recently, there has been much attention focused on possible functional implications of synaptic depression for computations such as gain control (Abbott et al., 1997), coincidence detection (Senn et al., 1998), and decorrelation (Goldman et al., 2002). Adaptation induced by slow intrinsic ionic currents of the spike generator is, however, also commonly observed in neurons and may enhance their response to highfrequency input (Nelson et al., 1997; French et al., 2001), mask low-intensity stimuli (Sobel and Tank, 1994; Wang, 1998), induce contrast adaptation (Sanchez-Vives et al., 2000), remove temporal correlations from the input (Wang et al., 2003), or affect network synchrony and rhythms (Crook et al., 1998; Ermen-

\footnotetext{
Received Nov. 24, 2004; revised Jan. 21, 2005; accepted Jan. 22, 2005.

This work was supported by a grant from the Canadian Institutes of Health Research to A.L. and L.M. We thank Joseph Bastian for providing the data for Figure $7 D$.

Correspondence should be addressed to Dr. Jan Benda, Institute for Theoretical Biology, Humboldt Universität, Invalidenstrasse 43, 10115 Berlin, Germany. E-mail: j.benda@biologie.hu-berlin.de.

A. Longtin's present address: Department of Physics, University of Ottawa, Ottawa, Ontario, K1N 6 N5 Canada. DOI:10.1523/JNEUROSCI.4795-04.2005

Copyright $\odot 2005$ Society for Neuroscience $\quad$ 0270-6474/05/252312-10\$15.00/0
}

trout et al., 2001; van Vreeswijk and Hansel, 2001; Fuhrmann et al., 2002). Because adaptation usually operates within complex neural circuits and on many different time scales (Fairhall et al., 2001; Baccus and Meister, 2002; Kohn and Whitsel, 2002), it has been difficult to determine how these different cellular mechanisms contribute to network-level computations [e.g., sensory adaptation (Chung et al., 2002; Castro-Alamancos, 2004) or contrast adaptation (Sanchez-Vives et al., 2000; Fairhall et al., 2001; Kim and Rieke, 2003) in the visual system]. There are few studies on the functional role of adaptation or synaptic depression within a behavioral context (Sobel and Tank, 1994; Cook et al., 2003; Luksch et al., 2004; Ronacher and Hennig, 2004).

Here, we examine a sensory system that exhibits prominent spike-frequency adaptation to constant input and in which the functional role of adaptation can be investigated in the context of natural and behaviorally relevant stimuli. By means of a detailed phenomenological characterization of the observed spikefrequency adaptation, we conclude that the underlying mechanism includes adaptation of spike generation. Furthermore, we demonstrate that spike-frequency adaptation permits the detection of transient weak communication signals in the presence of strong low-frequency signals by enhancing the response to faststimulus components.

The weakly electric fish Apteronotus leptorhynchus (brown ghost knifefish) generates a quasi-sinusoidal electric organ discharge (EOD) in the $700-1000 \mathrm{~Hz}$ range, the amplitude of which is encoded in the discharge of fast-adapting electroreceptors (Punits) (Bastian, 1981; Nelson et al., 1997). The electrosensory 
system is used for the detection of objects and prey as well as for communication. In the latter case, the superposition of the electric fields of two fish results in a beat, an EOD amplitude modulation (AM) with a frequency that is given by the frequency difference, $\Delta f$, of the two fish. Low-frequency beats $(|\Delta f|<50 \mathrm{~Hz})$ will evoke EOD modulations termed "chirps": small chirps are agonistic communication signals in which the frequency of the EOD is increased transiently (Zupanc and Maler, 1993).

In this study, we present in vivo recordings from electroreceptors showing that chirps can evoke a response that is significantly enhanced compared with the response to slower beats. Furthermore, using a recently developed universal model of adaptation (Benda and Herz, 2003), we successfully predict the firingfrequency response of electroreceptors to chirps. Thus, understanding the dynamics of adaptation is essential for explaining the measured data. It is the cutoff frequency of the high-pass filter induced by adaptation that separates slow beats, transmitted with low gain, from fast chirps that are transmitted with a larger gain.

\section{Materials and Methods}

Chirp recordings. For recordings of chirps as shown in Figure 1, fish were placed in a mesh between the stimulation electrodes. The fish was stimulated with sine waves of different randomly ordered frequencies -12 , $-10, \ldots, 10,12 \mathrm{~Hz}$ above the fish's EOD frequency at $20 \%$ contrast (10 $s$ long, $20 \mathrm{~s}$ pause). The spontaneously emitted chirps were detected as brief peaks $>20 \mathrm{~Hz}$ above baseline EOD frequency.

Electrophysiology. Data from nine adult A. leptorhynchus $(12-16 \mathrm{~cm})$ are used in this study. For surgical exposure of the electrosensory lateral line lobe (ELL), fish were anesthetized (MS-222; Sigma-Aldrich, St. Louis, MO). After surgery, fish were immobilized (Flaxedil; Sigma) and transferred into a tank $\left(28^{\circ} \mathrm{C}\right)$ where they were respired by a constant flow of oxygenated water through their mouth. Action potentials from single P-unit afferents were recorded routinely in vivo with sharp glass micropipettes $(100 \mathrm{M} \Omega)$ that were advanced into the ELL with a piezoelectric microdrive (Inchworm IW-711; Burleigh, Fishers, NY). The potential between the micropipette and the reference electrode, which was placed in the fish's neck muscle, was amplified (Axoprobe 1A; Axon Instruments, Union City, CA), bandpass filtered $(0.45-7 \mathrm{kHz}, \mathrm{PC} 1$; Tucker-Davis Technologies, Alachua, FL), and notch filtered at $60 \mathrm{~Hz}$ and the fish's EOD frequency (Ultra-Q Pro; Boehringer Mannheim, Willich, Germany). All experimental protocols were approved by the University of Ottawa Animal Care Committee.

The EOD unperturbed by the stimulus was recorded between the head and tail of the fish using two vertical carbon rods $(11 \mathrm{~cm}$ long, $8 \mathrm{~mm}$ diameter). The transdermal voltage constituting the stimulus picked up by the P-units was estimated by two silver wires coated with nail polish, 1 $\mathrm{cm}$ apart, placed perpendicular to the side of the fish. Both EOD voltages were amplified and low-pass filtered at $5 \mathrm{kHz}$ (2015F; Intronix, Bolton, Ontario, Canada). Stimuli were attenuated (PA4; TDT), isolated (model 2002; A-M Systems, Carlsborg, WA), and delivered by two stimulation electrodes (30-cm-long, 8-mm-diameter carbon rods) placed $10 \mathrm{~cm}$ on either side of the fish, parallel to its longitudinal axis. Two classes of stimuli were used: direct stimuli (sine waves to mimic the EOD of a second fish) and AMs. For the latter, the AM signal was multiplied with the fish's EOD (MT3; TDT). Note that with direct stimulation, the AMs measured from the EOD peaks are of the same size as the AMs measured from the EOD troughs: they equal the amplitude of the stimulus. In contrast, AM stimuli can result in unequally sized AMs if the EOD is asymmetric (often the peak is wider than the trough).

The extracellular potential, the EOD, the transdermal potential, and the attenuated stimulus were digitized at $20 \mathrm{kHz}$ with a 12-bit Multi-IO board (PCI-MIO-16E-4; National Instruments, Austin, TX) on an Intel Pentium IV 1.8 GHz Linux personal computer. Spike and EOD detection, stimulus generation and attenuation, and preanalysis of the data were performed on-line during the experiment within our OEL (Online Electrophysiology Laboratory) software.

Protocols and data analysis. Identified P-units (Bastian, 1981) were stimulated with artificial sinusoidal EOD waveforms with frequencies 5 , $10,20,30$, and $60 \mathrm{~Hz}$ above the fish's own EOD frequency, resulting in the same positive beat frequencies, $\Delta f$. The amplitude of the artificial EOD was chosen to be $\sim 20 \%$ of the amplitude of the fish's own EOD. Ten identical chirps were included into each stimulus as a Gaussian increase in EOD frequency, such that they were separated by at least one complete beat cycle and would appear at 10 different positions within the beat. The width of the chirps at $10 \%$ height was $14 \mathrm{~ms}$, and their size was 30,60 , 100,122 , or $153 \mathrm{~Hz}$ corresponding to phase advances $\Delta \varphi_{\mathrm{C}}=0.25,0.5$, $0.8,1.0$, and 1.25 (throughout this report, phases are defined modulo one). Each such stimulus was presented $10-20$ times. The actual position of a chirp within the beat was determined by measuring the phase shift between the stimulus and the fish's EOD, because even slight changes in the fish's EOD frequency make it impossible to accurately predict the position of the chirp within the beat cycle. Spike trains for the chirps were then sorted into 10 bins of different beat positions. The firing frequency was computed as the inverse interspike interval (ISI) averaged over the trials for every $0.5 \mathrm{~ms}$. We computed the gain in firing frequency as the maximum modulation depth of the firing-frequency response during the chirp divided by the modulation depth during the beat. To minimize noise, we smoothed the firing frequency with a $0.05 /|\Delta f|$ wide window before measuring the modulation depth during the beat. We also computed a gain for the stimulus as the modulation depth of the AM during the chirp divided by the modulation depth during the beat. The response gain shown in Figures 5 and 7 is the gain of the firing frequency divided by the gain of the stimulus. The latter was close to 1 in most cases.

Stimuli for measuring $f-I$ curves were composed of a 100 -ms-long preadaptation part of amplitude $I_{\text {pre }}$ immediately followed by a 150 -ms-long test stimulus of amplitude $I$. While holding $I_{\text {pre }}$ fixed, we varied $I$ randomly such that each $I$ was presented $6-16$ times. The cells completely adapted to the preadaptation stimulus. Therefore, the response evoked by the test stimulus revealed the properties of the cell being in a fixed state of adaptation. As the steady-state response, we measured the mean firing frequency within the last $100 \mathrm{~ms}$ of the test stimulus. The onset firing frequency was determined as the largest deviation from the response preceding the test stimulus within the first $30 \mathrm{~ms}$ of the response smoothed with a running average of $3 \mathrm{~ms}$ width. To parameterize $f-I$ curves, we fitted the Boltzmann function $f(I)=f_{\max } /(1$ $\left.+\exp \left(-k\left(I-I_{1 / 2}\right)\right)\right)$ to the data. The slope of the linear part of the $f-I$ curve is then given by $s=f_{\max } k / 4$.

Adaptation model. Benda and Herz (2003) derived a general model for the dynamics of spike-frequency adaptation (Eq. 1) from the known kinetics of various adaptation mechanisms acting on the spike generator ("encoder adaptation") [e.g., M-type currents, afterhyperpolarization (AHP)-type currents, and even slow recovery from inactivation of the fast sodium current]. If the dynamics of adaptation are slow compared with an ISI, then the much faster dynamics of the spike generator can be treated independently of the slower dynamics of the adaptation process. In the simplest case in which the stimulus is changing slowly within an ISI, the generation of spikes can be described by a simple mapping of the stimulus through the $f-I$ curve $f_{0}(I)$ of the neuron that returns the output firing frequency $f(t)$ as a function of the input stimulus $I(t)$ that varies with time $t$. Most mechanisms of encoder adaptation result in a change in an ionic current through the membrane of the neuron that is flowing in parallel with the ionic current $I$ constituting the input. Therefore, adaptation is acting subtractively on the input. In contrast, a divisive effect on the stimulus would be expected if adaptation is attributable to depressing synapses (Abbott et al., 1997; Tsodyks et al., 1998).

The adaptation current can be approximated by its temporal average $A$, because we assumed it to be slow compared with the spike generation. Averaging the dominant slow process of the adaptation mechanism (gating of the M-type current, removal of intracellular calcium for AHP-type currents) yields the following dynamics of the firing frequency $f(t)$ :

$$
\begin{gathered}
f=f_{0}(I-A), \\
\tau \frac{d A}{d t}=f_{\infty}^{-1}(f)-f_{0}^{-1}(f)-A .
\end{gathered}
$$

The onset $f-I$ curve $f_{0}(I)$ is the $f-I$ curve of the unadapted neuron that one gets by measuring the onset response to step-like stimuli of various in- 
tensities, $I$. The averaged adaptation current is subtracted from the input in Equation 1a and therefore shifts $f_{0}(I)$ to higher input intensities. Because adaptation currents are activated by the generated spikes, the dynamics of adaptation is driven by the output firing frequency. For an expression of the steady-state value of $A$, we used the steady-state $f-I$ curve [see Fig. 6 for an example of $f_{0}(I)$ and $\left.f_{\infty}(I)\right]$. In particular, the state of adaptation $A(t)$ in Equation $1 \mathrm{~b}$ is driven by the horizontal distance $f_{\infty}^{-1}(f)-f_{0}^{-1}(f)$ between the onset and steady-state $f-I$ curve at the firing frequency $f$.

Other plausible adaptation mechanisms, acting, for example, on the transduction of a physical stimulus in a receptor neuron (Gollisch and Herz, 2004), could be driven by the input stimulus I directly. In this case, the dynamics for $A$ is governed by the following:

$$
\tau \frac{d A}{d t}=I-f_{0}^{-1}\left(f_{\infty}(I)\right)-A
$$

where $I-f_{0}^{-1}\left(f_{\infty}(I)\right)$ is the distance of the two $f-I$ curves at the steady-state firing frequency $f_{\infty}(I)$. In contrast to the corresponding term for output-driven adaptation in Equation $1 \mathrm{~b}$ that depends on the actual firing frequency, this term is constant for a constant stimulus $I$.

In the special case of linear $f-I$ curves, the model for output-driven adaptation Equation 1 is linear and constitutes a high-pass filter with gain as follows:

$$
g\left(f_{c}\right)=f_{\infty}^{\prime} \sqrt{\frac{1+\left(2 \pi f_{c} \tau_{\text {eff }} f_{0}^{\prime} / f_{\infty}^{\prime}\right)^{2}}{1+\left(2 \pi f_{c} \tau_{\text {eff }}\right)^{2}}},
$$

where $f_{0}^{\prime}$ and $f_{\infty}^{\prime}$ are the slopes of the $f-I$ curves, and $f_{c}$ is the frequency component of the stimulus $I(t)$. The effective time constant of adaptation $\tau_{\text {eff }}=\tau f_{\infty}^{\prime} / f_{0}^{\prime}$ can be observed as the exponential decay of the firing frequency evoked by a steplike stimulus. It is smaller than the adaptation time constant $\tau$ describing the dynamics of the adaptation mechanism. Note that for input-driven adaptation, $\tau_{\text {eff }}$ equals the adaptation time constant $\tau$ in Equation 2.

Equations 1-3 describe the effect of adaptation only. However, if stimulus fluctuations are fast compared with an ISI $(\approx 1 / f)$, the low-pass filter effect of spikes has to be taken into account by specifying a model for spike generation (Benda and Herz, 2003). The perfect integrate-and-fire neuron,

$$
\frac{d \Psi}{d t}=f(t)
$$

requires no additional parameter and is the canonical model for the dynamics on a limit cycle, as emerging by suprathreshold stimulation. Whenever the phase $\Psi$ reaches the value $1, \Psi$ is reset to zero, and a spike is elicited. From these spikes, a firing frequency can be calculated as 1/ISI and compared with the experimentally measured one.

The two models Equations 1 and 2 in combination with Equation 4 are fully described by the onset and steady-state $f-I$ curves of the neuron, $f_{0}(I)$ and $f_{\infty}(I)$, and by the adaptation time constant $\tau$. Therefore, the firing-frequency responses of an adapting neuron to time-varying stimuli can be predicted after determining the $f-I$ curves of the neuron and adaptation time constant with step stimuli.

\section{Results}

\section{Chirps: signal characteristics}

Small chirps (also known as type II chirps) are brief events in which a fish raises the frequency of its EOD by $30-150 \mathrm{~Hz}$ (the
C

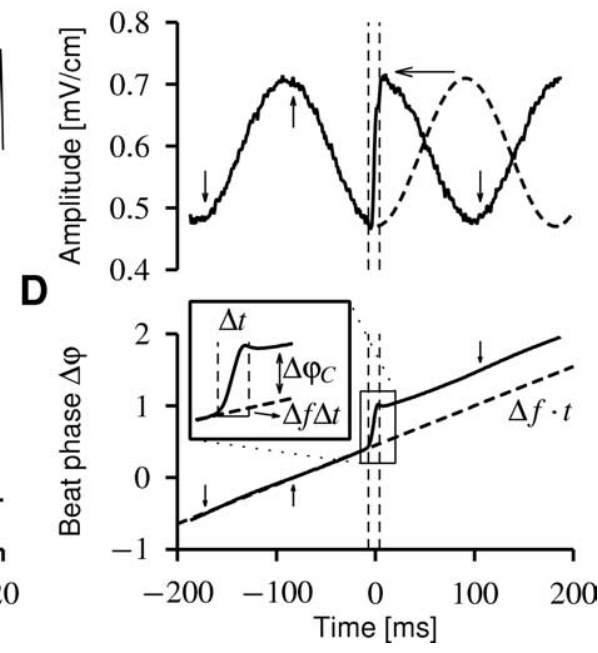


A

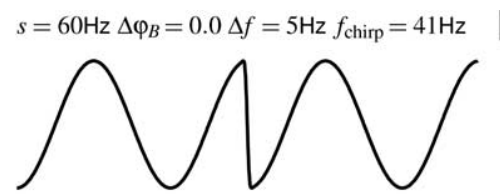

B
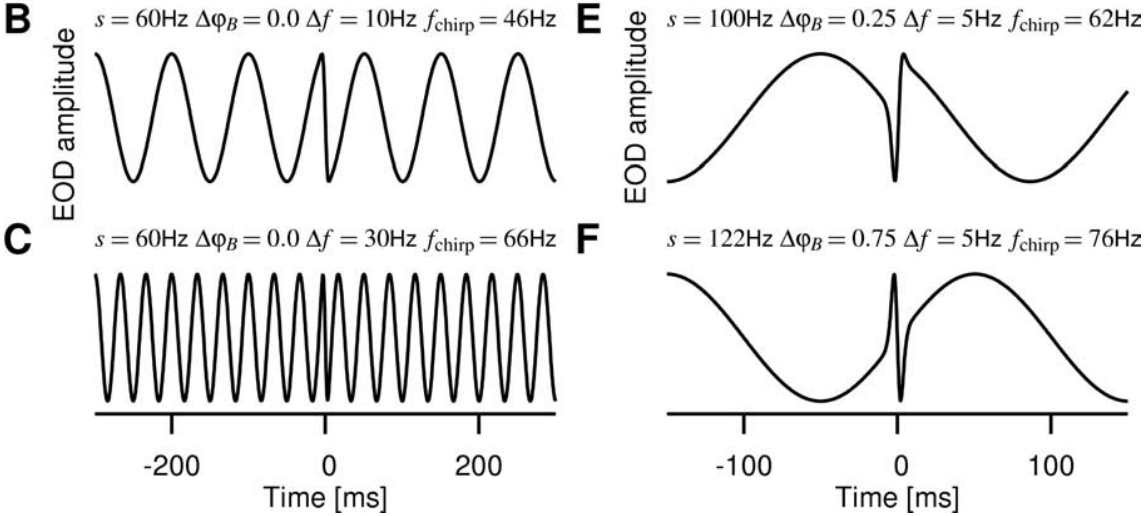

Figure 2. Stimuli generated by chirps. EOD AMs resulting from $\Delta t=14$-ms-wide chirps of different peak frequency increases (size s of the chirp) centered around time 0 on various positions $\Delta \varphi_{B}$ within the beat of frequency $\Delta f$, as indicated, are shown. The traces were generated assuming a Gaussian frequency increase in the EOD during the chirp according to $A(t)=\cos (2 \pi \Delta \varphi(t))$, where $\Delta \varphi(t)=\Delta \varphi_{\mathrm{B}}+\Delta f t+s \sigma \int_{-\infty}^{t / \sigma} \exp \left(-z^{2}\right) d z$ is the phase of the beat as the time integral of the frequency difference of the two EODs and $\sigma=\Delta t /(2 \sqrt{\ln 10})$. These artificial EOD AMs are very similar to AMs resulting from real chirps [compare with Zupanc and Maler (1993) and Fig. 1C ]. $f_{\text {chirp }}$ is an estimate of the stimulus frequency during the chirp as given in Equation 5. A-C, A $60 \mathrm{~Hz}$ chirp advancing the beat by one-half of a cycle $\left(\Delta \varphi_{c}=0.5\right)$ on different beat frequencies, $\Delta f=5,10$, and $30 \mathrm{~Hz}$. The chirp is located at the peak of the beat and thus produces a sudden downstroke in EOD amplitude, which, however, is less salient at high beat frequencies (C). D, Same chirp as in $\boldsymbol{A}$ but one-half of a cycle later in the beat, therefore starting in the trough of the beat and producing an upstroke. $E, A 100 \mathrm{~Hz}$ chirp advancing the beat by $\Delta \varphi_{\mathrm{C}}=0.8$ cycles at the zero-crossing of the beat-sine wave. $\boldsymbol{F}$, After a $122 \mathrm{~Hz}$ chirp $\left(\Delta \varphi_{C}=1\right)$, the beat continues without a phase shift. The fish emit chirps on every position within the beat (data not shown).

of width $\Delta t$ is the phase shift $\Delta f \Delta t$, attributable to the beat alone plus the phase shift $\Delta \varphi_{C}$ of the chirp. Dividing by $\Delta t$ yields the slope of $\Delta \varphi$ and thus the characteristic frequency $f_{\text {chirp }}$ of the AM during the chirp:

$$
f_{\text {chirp }}=\Delta f+\Delta \varphi_{\mathrm{C}} / \Delta t
$$

The second term is in the order of $\sim 70 \mathrm{~Hz}\left(\Delta \varphi_{\mathrm{C}} \approx 1, \Delta t \approx 14 \mathrm{~ms}\right)$; it ranges from $18 \mathrm{~Hz}\left(\Delta \varphi_{\mathrm{C}} \approx 0.25\right)$ to $110 \mathrm{~Hz}\left(\Delta \varphi_{C} \approx 1.5\right)$. Therefore, as shown in Figure 2, chirp-induced AMs are faster than the beat AMs that typically elicit them $(|\Delta f|<50 \mathrm{~Hz}$ ) (Bastian et al., 2001), but the relative difference in time scales is reduced for higher beat frequencies proportional to $1 /|\Delta f|$ (Fig. $2 C$ ).

\section{P-unit response}

The response of a single P-unit stimulated with the superposition of the fish's own EOD and an artificial stimulus mimicking a second fish's EOD with chirps is shown in Figure 3A. As is known, the firing-frequency response (Fig. $3 B$ ) closely follows the slow beat (Bastian, 1981; Nelson et al., 1997). In the example shown, the response ranges from $50 \mathrm{~Hz}$ in the troughs to $250 \mathrm{~Hz}$ on the peaks, a $200 \mathrm{~Hz}$ firing frequency modulation. The chirp in this example produces one-half of an EOD cycle of phase advance in the trough of the beat, resulting in a fast upstroke in EOD amplitude (Fig. 3C). This signal evokes a clearly enhanced response of up to $420 \mathrm{~Hz}$, overshooting the response to the beat peaks almost by a factor of 2 .

Chirps are frequency modulations produced by one fish that are converted into AMs by the superposition of the EOD of another fish. To confirm whether the enhanced response of the P-units to chirps is solely caused by the resulting AM, we addi- tionally stimulated the fish with an AM similar to that generated by the chirp, but without the frequency modulation of a real chirp (see Materials and Methods) (Fig. 3D-F). The averaged mean difference between the firing-frequency response to the real chirp and the pure AM was $59 \pm$ $50 \mathrm{~Hz}(n=257$ chirps $)$, within $\pm 50 \mathrm{~ms}$ around the chirp. This makes the two firing frequencies indistinguishable ( $\chi^{2}$ test; $p=0.66 \pm 0.47)$.

Additional examples of $\mathrm{P}$-unit responses to chirps of different sizes at various beat positions and beat frequencies are shown in Figure 4. Chirp-induced downstrokes in EOD amplitude evoke a strongly reduced firing frequency or even a period of silence after the chirp (Fig. 4A,C). Upstrokes evoke strongly enhanced firing frequencies (Fig. 4B,C). Especially large chirps with phase shifts $>0.5$ produce both upstrokes and downstrokes (Fig. $2 E, F)$, often resulting in a very low response followed by a very high response, as in Figure $4 C$, and vice versa. However, at higher beat frequencies, the response to chirps is just as large as the response to the beat (Fig. $4 D$ ).

We analyzed the responses of 36 P-units to 1208 chirps altogether. Each of these chirps had one of five different sizes $(30,60,100,122$, and $153 \mathrm{~Hz}$ peak frequency increase corresponding to phase shifts of $0.25,0.5,0.8,1.0$, and 1.25) on five different beat frequencies $(5,10,20,30$, and $60 \mathrm{~Hz})$ and occurred on 10 different beat positions. Thus, only a small subset of all possible combinations of chirp sizes and beat frequencies was measured in each unit. On average, 13.5 trials were measured for each chirp. We assumed that the chirps are mainly coded by firing frequency and that the detectability of a chirp within a beat can be estimated by the response gain, which basically is the maximum modulation depth of the firing-frequency response during the chirp divided by its modulation depth during the beat (see Materials and Methods). The response gain did not show a significant dependence on chirp size $(r=0.03 ; p=0.3 ; n=1177)$ or beat position $(r=0.02$; $p=0.5 ; n=1208)$.

However, as suggested in Figure 4, beat frequency had a large effect on response gain (Fig. 5). For the $5 \mathrm{~Hz}$ beat, the response gain was, on average, $2.0 \pm 0.7$, thus enhancing the firing frequency twofold compared with the beat response. The response gain monotonically decreases as beat frequency increases. At 30 $\mathrm{Hz}$, it is already down at $1.08 \pm 0.20$, meaning that the response to the beat is now as strong as the response to the chirp. These data suggest that there is a cutoff frequency in the system between 20 and $30 \mathrm{~Hz}$ that separates slow beats from chirps and fast beats, thus defining two ranges of signal time scales: slow beats on which chirps are detectable as an enhanced firing-frequency response and fast beats on which the P-unit firing-frequency response to chirps becomes invisible.

\section{Spike-frequency adaptation and high-pass filtering}

The data could be explained with a high-pass filter that transmits high-frequency stimuli such as chirps with a much larger gain 
than low-frequency stimuli such as beats. This may be accounted for by the known spike-frequency adaptation observed in P-units (Hagiwara and Morita, 1963; Bastian, 1981; Xu et al., 1996; Nelson et al., 1997). To test this hypothesis, we used a general model for the firing-frequency dynamics of an adapting neuron that was recently derived by Benda and Herz (2003) (see Materials and Methods). The model is completely characterized by the onset and steady-state $f-I$ curves as well as the effective time constant of adaptation, all of which can be easily determined from the response of the neuron to step-like stimuli. Therefore, the model does not require any biophysical specification of the adaptation process. Adaptation of $\mathrm{P}$-units, which are afferent fibers that innervate numerous tuberous electroreceptors (Bennett et al., 1989), might be attributable to adaptation of the transduction mechanism within the electroreceptors, depression of their synapses onto the afferents, or adaptation of the spike generator in the afferent. Within the model framework, these three fundamentally different adaptation mechanisms can be distinguished based on the stepresponse data.

We measured responses to step-like increases and decreases in the fish's EOD amplitude in 18 cells, using the AM-type stimulation as described in Materials and Methods. Figure $6 A-C$ shows three examples of firing-frequency $(f)$ responses to various intensities, $I$, of a constant stimulus switched on at $t=0$. The initial onset-response $f_{0}$ of the firing frequency to the step stimulus reflects properties of the neuron in a fixed state of adaptation $A$. The steady-state response $f_{\infty}$ reveals information about the maximum adaptation strength at a given stimulus intensity $I$. Measuring both quantities for different stimulus intensities results in the onset $f-I$ curve $f_{0}(I)$ and the steadystate $f-I$ curve $f_{\infty}(I)$, as shown in Figure $6 D$. Both types of $f-I$ curves are linear over a wide range of firing frequencies. Whereas the steady-state $f-I$ curve spans the entire range of tested EOD amplitudes (more than $\pm 20 \%$ of the EOD baseline amplitude), the onset $f-I$ curve is much steeper and saturates at the EOD frequency at which the neuron fires a spike at each EOD cycle and goes to zero for lower amplitudes. This and saturating responses such as the one shown in Figure $6 B$ is consistent with an adaptation process that must act at or before the generation of spikes in which the saturation is introduced.

Adaptation could have a subtractive or divisive effect on the onset $f-I$ curve. To distinguish between these two possibilities, we measured the onset $f-I$ curve for different levels of adaptation by increasing or decreasing the EOD amplitude to some value $I_{\text {pre }}$ before measuring the response for different intensities, $I$. In all cells, the onset $f-I$ curves for the different adaptation levels are only shifted along the intensity axis (Fig. $6 F$ ). Dividing the slopes of each pair of $f-I$ curves of a cell, such that the slope of the $f-I$ curve measured at the smaller preadaptation intensity $I_{\text {pre }}$ is divided by the one at a larger preadaptation intensity, and averaging over all pairs results in $1.02 \pm 0.21$, which is indistinguishable from $1(n=22 ; p=0.66 ; t$ test $)$, showing that the adaptation
Real Chirp

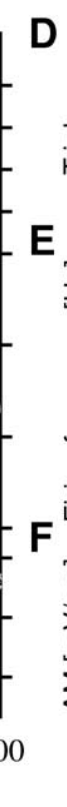

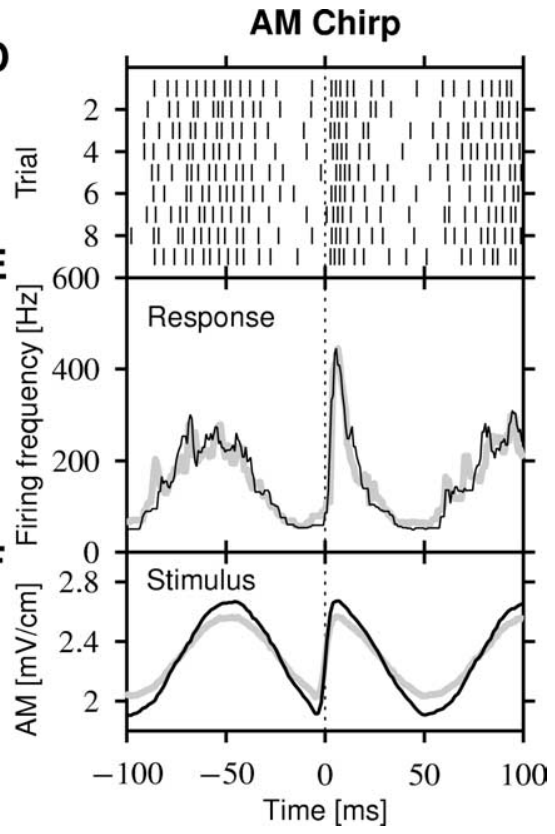

Figure 3. P-unit response to a chirp. The example shows a $60 \mathrm{~Hz}$ chirp at the trough of a $\Delta f=10 \mathrm{~Hz}$ beat. $A$, The spike trains e). The gray line is the prediction of the spike-frequency adaptation model Equations 1 and 4 . $C$, The relevant stimulus for the B). $\boldsymbol{F}$, The averaged stimulus for the AMs (gray line) and the real chirps (black line; same as in $\boldsymbol{B}$ ) differ in modulation depth waveform (see Materials and Methods).

process under investigation acts purely subtractively on the input. This suggests a weak influence of depressing synapses, because these would have a divisive effect on the input (Abbott et al., 1997; Tsodyks et al., 1998). Although, ultimately, only appropriate experiments can rule out the synapses as the locus of adaptation, the important finding is that adaptation in the electroreceptors is subtractive and not divisive.

The dynamics of adaptation can be driven either by the output spikes per firing frequency or the input stimulus directly (Eqs. 1, 2 ). The distinction between these different dynamics is more subtle, because for linear parts of the $f-I$ curves, they result in exactly the same equations for the firing frequency. Knowing the onset and steady-state $f-I$ curves (parameterized with a Boltzmann function), the only free parameter for both types of adaptation dynamics is the adaptation time constant $\tau$. Fitting the models to the step-response data, we find for the output-driven model (Eq. 1) (Fig. 6A-C, gray lines) a slightly, although not significantly, better performance than for the input-driven model Equation 2. The root mean squared difference between the firing frequency of the models and the measured firing frequency is $\sigma_{\text {diff }}=31 \pm 25$ $\mathrm{Hz}$ for output-driven adaptation and $\sigma_{\text {diff }}=32 \pm 36 \mathrm{~Hz}$ for input-driven adaptation $(n=639)$.

The negative correlation of ISIs that is observed in P-unit baseline activity (Hagiwara and Morita, 1963; Chacron et al., 2000; Ratnam and Nelson, 2000) can only be explained by spikedriven adaptation processes, because we already ruled out depressing synapses that also can induce negative ISI correlations (Goldman et al., 2002). The negative ISI correlations of the P-unit response can be easily reproduced by adding a simplified spike- 
A

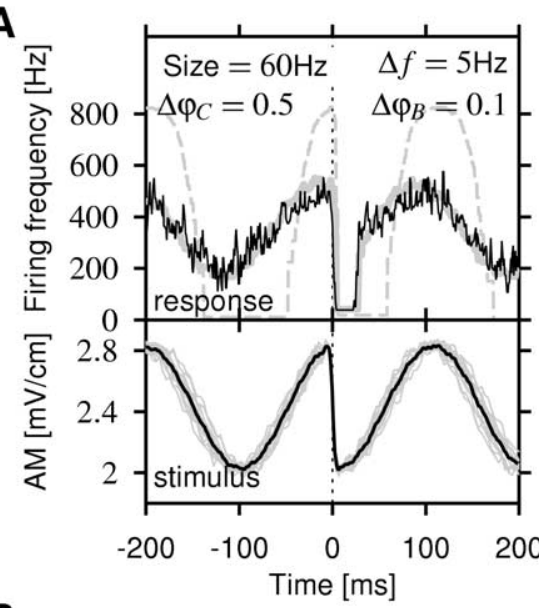

B

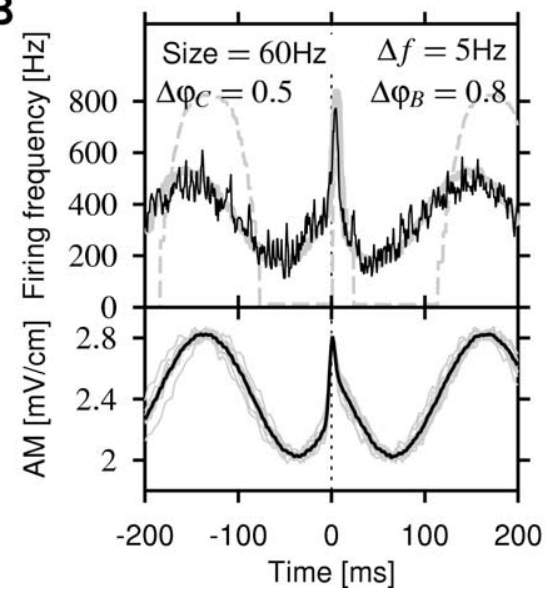

C

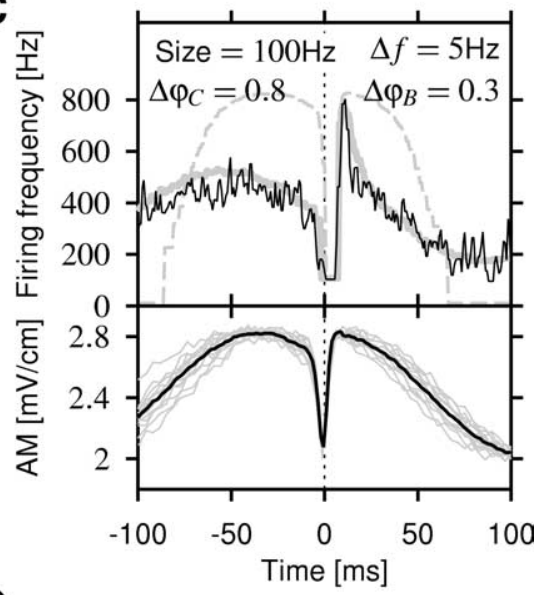

D

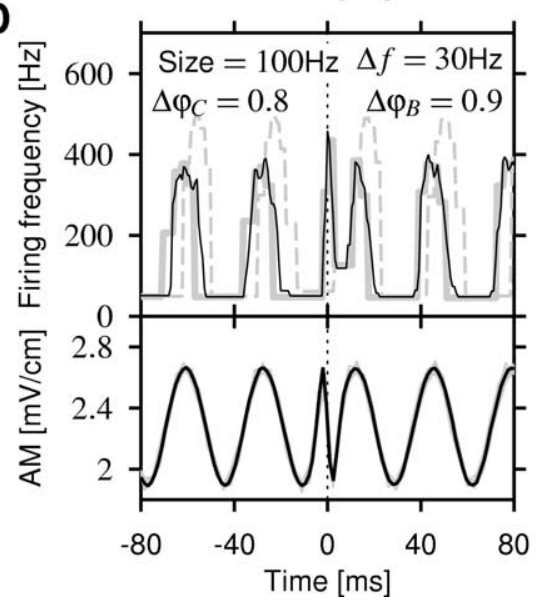

Figure 4. Firing-frequency responses (top panels; black line) to various-sized chirps generating a phase shift, $\Delta \varphi_{c}$, on a beat with frequency $\Delta$ fat beat position $\Delta \varphi_{B}$ as indicated. $A-C$, Recordings from a single P-unit with $p=0.42$ (baseline firing rate, 346 $\mathrm{Hz}$; EOD frequency, $826 \mathrm{~Hz}$ ) are shown. The corresponding AMs of the transdermal EOD are shown in the bottom panels (individual stimuli, gray; averaged stimulus, black). The model prediction (top panels; gray solid line) closely follows the measured firing frequency (black line), whereas the prediction with the onset $f-I$ curve alone does not describe the data (dashed gray line). A, A 60 $\mathrm{Hz}$ chirp occurring around the top of a $5 \mathrm{~Hz}$ beat generates a fast downstroke in $\mathrm{EOD}$ amplitude. This causes the neuron to entirely stop spiking. In contrast, the neuron still fires at the troughs of the beat. Note that the firing frequency cannot equal zero, because it is measured as the inverse ISI. Periods of silence appear as straight horizontal lines. $\boldsymbol{B}$, Chirps at around the trough of the beat generate fast upstrokes, causing a much stronger response than the one during beat peaks. $C$, A larger $100 \mathrm{~Hz}$ chirp produces a downstroke immediately followed by an upstroke. This results in a short pause followed by a strong peak in the firing-frequency response. Note the enlarged time axis. $\boldsymbol{D}, \mathrm{A} 100 \mathrm{~Hz}$ chirp on a faster $30 \mathrm{~Hz}$ beat recorded from a different unit (same unit as in Fig. 3). The response to the beat is almost as large as the response to the chirp.

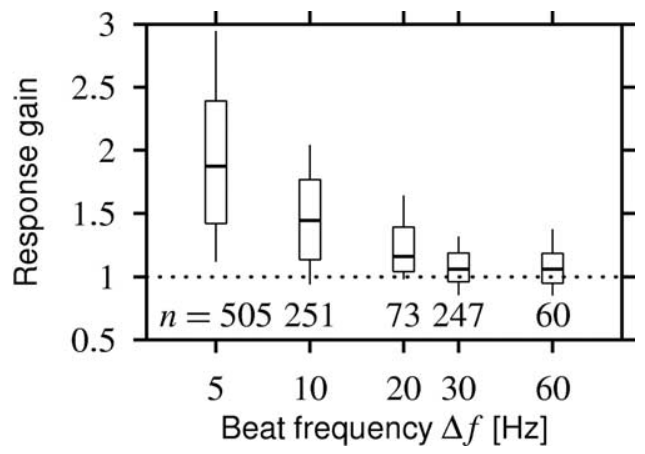

Figure 5. Response gain as a function of beat frequency $\Delta f$ for all 1208 chirp responses measured. The horizontal line within the boxes represents the median, the box includes the 2 nd and 3 rd quartiles, and the ends of the vertical lines mark the 1st and 9th deciles. Chirps evoke a response that is larger than the response to the beat (response gain $>1$ ) if the beat frequency is smaller than $\sim 30 \mathrm{~Hz}$.

driven adaptation current with a time constant of $42 \mathrm{~ms}$ (see below) to a perfect integrate-and-fire neuron [as proposed by Benda and Herz (2003), their Eq. 6.5] that is directly driven by the EOD with additive noise (data not shown). This additionally favors the hypothesis that the observed spike-frequency adaptation is caused by ionic currents acting on the spike generation, possibly mediated by Kv3.1 channels (Chacron et al., 2001). Thus, all of the characteristics of the adaptation model Equation 1 are met. Note that the model was originally derived for current stimuli that are directly applied to the spike generator, whereas here we only take the AM separated from the EOD as the stimuli. We show how to resolve this issue in the Appendix.

The adaptation time constants $\tau$ obtained by fitting the output-driven model Equation 1 to the step-response data are independent of stimulus intensity $(r=$ $-0.05 \pm 0.54 ; p=0.24 \pm 0.31$; mean \pm SD from 38 cells with each 10-29 measured $\tau-I$ pairs) (Fig. $6 E$ ), thus confirming the assumption of the model of $\tau$ being constant. The time constants are also independent of the baseline firing rate of the P-units $(r=-0.23 ; p=0.17)$ or $p$ value (baseline firing rate divided by EOD frequency; $r=-0.14 ; p=0.4)$ and averaged to $42 \pm 17 \mathrm{~ms}$ ( $n=38$ cells $)$.

The adaptation time constant describes the dynamics of the adaptation mechanism. It is usually larger than the effective time constant of adaptation that is obtained by fitting a single-exponential function $\left[f(t)=\left(f_{0}-f_{\infty}\right) \exp \left(-t / \tau_{\text {eff }}\right)+f_{\infty}\right]$ to the time course of the firing frequency evoked by step-like stimuli (Fig. 6A-C, dotted line), the reason being that in output-driven adaptation, the state of adaptation relaxes toward a value that is not constant and set by the stimulus but rather depends on the current firing frequency. The effective time constant of adaptation is independent of stimulus intensity $(r=$ $0.01 \pm 0.38 ; p=0.38 \pm 0.29$; mean \pm SD from 38 cells with each $5-28$ successfully measured $\tau_{\text {eff }}-I$ pairs) (Fig. $6 E$ ) independent of the baseline firing frequency of the cells $(r=-0.27 ; p=0.09)$ or $p$ value $(r=-0.26 ; p=0.12)$ and averages to $6.9 \pm 2.0 \mathrm{~ms}(n=$ 38 cells), which is one-sixth of the value of $\tau$. The two time constants $\tau$ and $\tau_{\text {eff }}$ are related by the relative slopes $f_{0}^{\prime}$ and $f_{\infty}^{\prime}$ of the onset and steady-state $f-I$ curves via $\tau_{\text {eff }}=\tau f_{o}^{\prime} / f_{0}^{\prime}$, if the $f-I$ curves are approximately linear (Benda and Herz, 2003). Indeed, we find for the relative slopes $f_{0}^{\prime} / f_{\infty}^{\prime}$ a value of $6.0 \pm 1.6$.

The high-pass filter Equation 3 induced by spike-frequency adaptation can be characterized by a cutoff frequency that separates slow stimuli that are transmitted with low gain from fast stimuli that are transmitted with a larger gain (Fig. 7A). The cutoff frequency $f_{\text {cutoff }}=\left(2 \pi \tau_{\text {eff }}\right)^{-1}$ is determined by the effective time constant of adaptation (Benda and Herz, 2003). From our data, we obtain $f_{\text {cutoff }}=23 \pm 7 \mathrm{~Hz}$. However, because the high- 
pass filter Equation 3 is just a single-pole filter, it has no sharp cutoff. The gain raises rather gradually from low to high values over the range of stimulus frequencies considered in this study.

\section{Model prediction for beats and chirps}

Having measured the onset $f-I$ curve, the steady-state $f-I$ curve, and the adaptation time constant for a given cell, the adaptation model Equation 1 is defined completely. In combination with the perfect integrator Equation 4 for simulating a spike generator (see below and Materials and Methods), we can now predict the firing-frequency response of the same cell to an entirely different set of stimuli, namely beats and chirps, without any additional parameter fitting. For this purpose, we feed the measured AM of the EOD evoked by the chirp as the stimulus $I(t)$ into the model Equations 1 and 4. As Figures 3 and 4 show, the prediction of the model closely follows the firing-frequency response to the beats as well as the chirps $\left(\chi^{2}\right.$ test; $\left.p=0.51 \pm 0.49 ; n=635\right)$. The root mean squared difference $\sigma_{\text {diff }}$ between the predicted and the measured firing frequency within $\pm 50 \mathrm{~ms}$ around each chirp is $56 \pm 36 \mathrm{~Hz}$, on average. Comparing this error to the modulation depth of the response during the beat results in a prediction error of $24 \pm 8 \%$. On the contrary, neglecting adaptation and just using the onset $f-I$ curve $\left[f=f_{0}(I)\right]$ obviously fails to predict the firing frequency (Fig. 4, dashed gray lines). The good performance of the adaptation model shows that spikefrequency adaptation is sufficient for explaining the firing-frequency response of the electroreceptors to chirps.

What happens is that the adaptation strength follows low-frequency beats (i.e., in terms of the model, the onset $f-I$ curve is shifted according to the stimulus to lower and higher intensities, which results in firing frequencies that are close to the ones given by the steady-state $f-I$ curve). An AM generated by a chirp, however, is too fast for the adaptation dynamics to follow; the onset $f-I$ curve will practically stay where it was right before the chirp. If the chirp occurs at the peak of the beat, then the neuron is strongly adapted (i.e., the onset $f-I$ curve is shifted to high stimulus intensities), and if the sudden downstroke of the produced AM easily gets below the threshold of the $f-I$ curve, the neuron stops firing. If the chirp occurs during the trough of the beat, then the neuron is very sensitive, because its onset $f-I$ curve is shifted to low stimulus intensities. The sudden upstroke caused by the chirp thus evokes very high firing frequencies that then rapidly adapt back to values given by the steady-state $f-I$ curve.

Using the gain $g\left(f_{\mathrm{c}}\right)$ Equation 3 of the high-pass filter of the linearized adaptation model with the mean values $\tau_{\text {eff }}=7 \mathrm{~ms}$ and their shape.

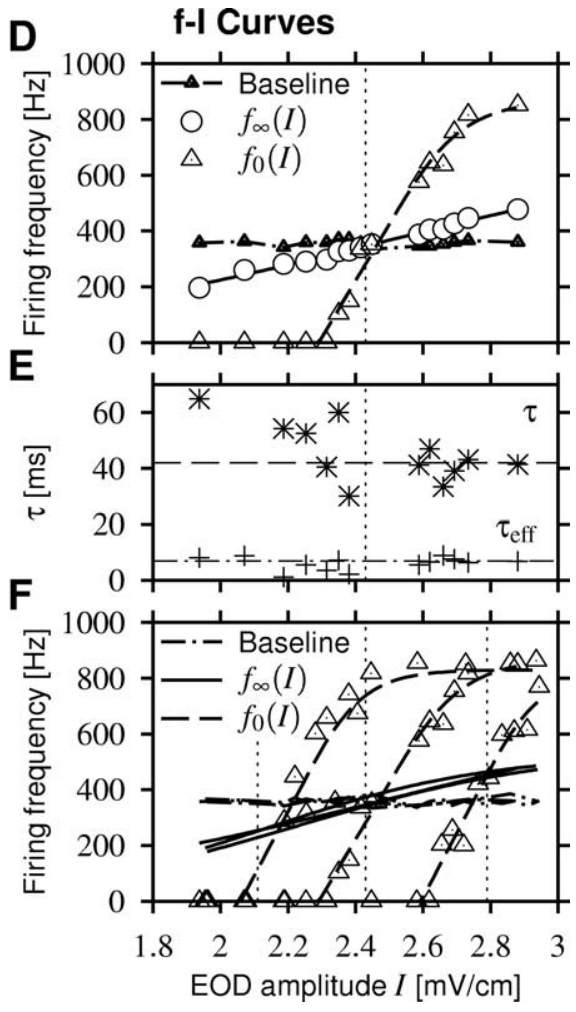

Figure 6. Firing frequency-intensity curves ( $f-I$ curves) measured in the same cell as in Figure $4 \boldsymbol{A}-\boldsymbol{C}$. First, the cell was adapted to some EOD amplitude $I_{\text {pre }}$ higher, lower, or equal to the EOD baseline amplitude $(2.43 \mathrm{mV} / \mathrm{cm})$ to prepare the cell in firing-frequency response to the second part of the stimulus are shown in $\boldsymbol{A}-\boldsymbol{C}$ (black solid lines). An exponential fit (dotted line; clipped at the maximum response) measured the effective time constant of adaptation $\tau_{\text {eff }}$, and a fit of the computed response 0 step responses describes the data sufficiently well but, in contrast to the adaptation model Equation 1, does not define a dynamics

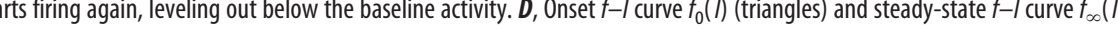

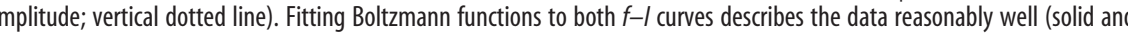
measured during 100 ms before applying the preadapting stimulus. $\boldsymbol{E}$, The effective time constant $\tau_{\text {eff }}(+)$ and the adaptation for three values of $I_{\text {pre }}$ (vertical dotted lines) of the preadaptation stimulus and thus three different states of adaptation. By finition, the steady-state $f-I$ curves (solid lines) and the baseline firing frequencies (dashed dotted line) are independent of $I_{\text {pre }}$ In contrast, the onset $f-I$ curves (triangles, data; dashed lines, fit) are shifted by adaptation along the intensity axis while keeping

$f_{0}^{\prime} / f_{\infty}^{-1}=6$, we can predict the response gain as the gain of the chirp divided by the gain of the beat, as follows:

$$
\text { response gain } \approx g\left(\left|\Delta f+\Delta_{\varphi C} / \Delta t\right|\right) / g(|\Delta f|) \text {, }
$$

where we used Equation 5 for an estimate of the AM frequency during a chirp. Chirps are high-frequency events with respect to the cutoff frequency of the high-pass filter. Therefore, their gain is maximal and approximately independent of the beat frequency $\Delta f$ for positive $\Delta f$. Dividing by the gain of the beat results in a function that gradually decreases with increasing $|\Delta f|($ Fig. $7 B)$. It reproduces the decay of the response gain data with increasing $\Delta f$ qualitatively but drastically overestimates the observed gain (Fig. $7 C$, circles), especially at low beat frequencies.

In addition to the response gain estimate Equation 6, which assumes linear $f-I$ curves, the model Equation 1 takes the satura- 
A

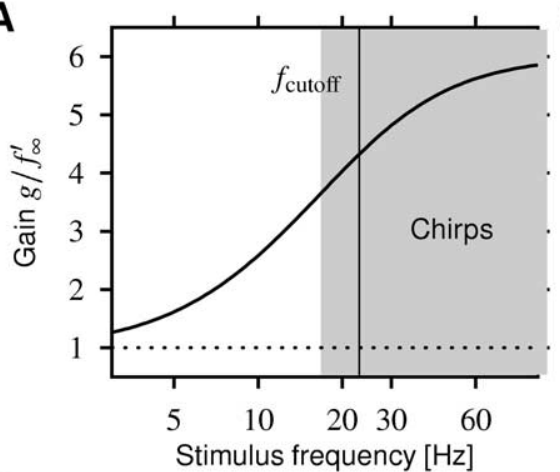

B

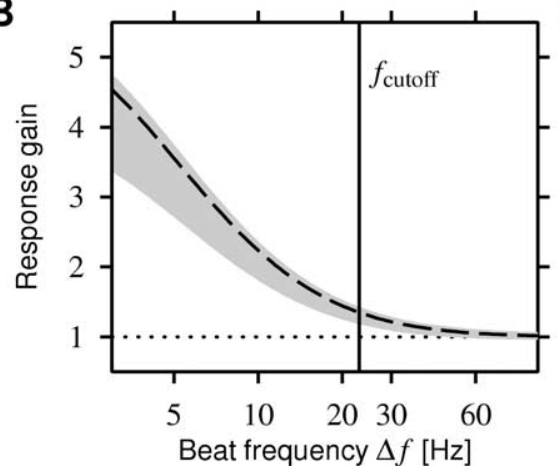

C

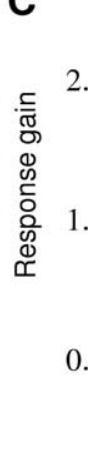

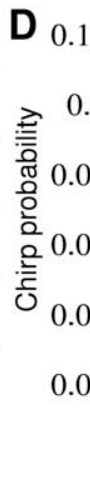

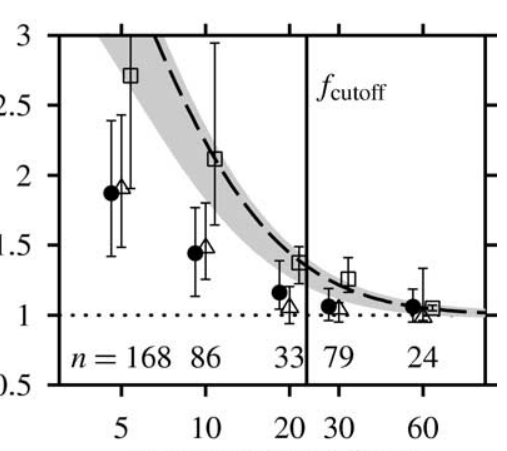

Beat frequency $\Delta f[\mathrm{~Hz}]$

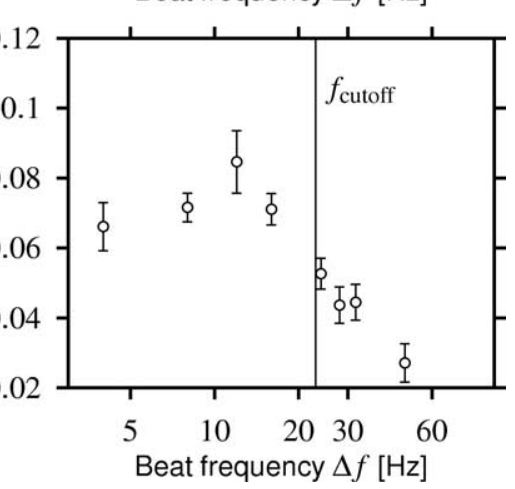

Figure 7. Comparison of the response-gain data with model predictions and behavior. $\boldsymbol{A}$, The gain Equation 3 of the high-pass filter generated by adaptation (solid line) as a function of stimulus frequency. The averaged value of the measured effective adaptation time constants sets the cutoff frequency $f_{\text {cutoff }}$ of the gain function to $23 \mathrm{~Hz}$ (vertical line in all panels). Chirps are high-frequency signals (gray area) that are transmitted with a high gain. $\boldsymbol{B}$, The response gain Equation 6 as a function of positive beat frequencies $\Delta$ f estimated from the high-pass filter shown in $\boldsymbol{A}$. The dashed line is the response gain for 14-ms-wide chirps that generate a phase shift of 1 , and the gray area is for phase shifts ranging from 0.25 to 1.5 . $\boldsymbol{C}$, The response gain from $\boldsymbol{B}$ (dashed line and gray area) explains the decay of the observed response gain only qualitatively (filled circles; median with 2nd and 3rd quartiles). For the 18 cells in which $f-I$ curves were measured, we computed the response gains as predicted by the models. Using the adaptation model Equation 1, thus taking the saturating $f-l$ curves into account, does not improve the match (squares). However, the additional low-pass filter properties introduced by spikes, modeled using the perfect integrator Equation 4, reduce the predicted response gains significantly (triangles), resulting in a much better match to the actually observed data. The variability of the response- gain data can be mainly attributed to the different sized chirps (compare error bars to the width of the gray area). D, The probability of a male fish emitting chirps as a function of beat frequency as reported by Bastian et al. (2001), their Fig. 3 A.

tion of the $f-I$ curves into account. The response gain computed from the prediction of the model (Fig. $7 C$, squares) is, however, still very similar to the linear estimate.

The adaptation model Equation 1a maps the fast transients of the chirp stimulus directly into a firing frequency via the onset $f-I$ curve. However, as pointed out by Benda and Herz (2003) (their Eq. 6.3), during each ISI, a suprathreshold-driven spike generator integrates over the stimulus along its limit cycle. This temporal averaging results in low-pass filtering that can have a substantial effect on the response to stimulus transients that are short compared with an ISI (especially at periods of silence as in Fig. 4A). For predicting the firing-frequency response, we already used the perfect integrate-and-fire neuron Equation 4 to generate spikes and thus to account in the simplest possible way for the low-pass filter effect. The response gain calculated from the predictions (Fig. 7C, triangles) indeed matches the observed values well (Fig. $7 C$, circles).

\section{Discussion}

The example we have presented here emphasizes the general functional role of high-pass filter properties that result from any kind of spike-driven adaptation. We have demonstrated how adaptation in electroreceptor afferents of the weakly electric fish $A$. leptorhynchus enhances the response to small but fast communi- cation signals (i.e., the chirps) by temporally separating them from slower background signals (i.e., the beats). The stimuli generated by small chirps boil down to a sudden and short increase in AM frequency of the EOD by $\sim 18-110 \mathrm{~Hz}$ on top of a beat pattern of arbitrary frequency. From the effective time constant $\tau_{\text {eff }}=7 \mathrm{~ms}$, measured from the exponential decay of the firingfrequency response of the electroreceptor afferents to constant stimuli, we conclude that the cutoff frequency of the generated highpass filter is at $f_{\text {cutoff }}=23 \mathrm{~Hz}$. This cutoff frequency defines a time scale that separates slow from fast stimuli. Chirps on beat frequencies below that frequency give rise to an enhanced response, whereas on higher beat frequencies, the response to the beat is about as strong as the one to the chirp. Note that this result is independent of the phase shift (Fig. 1C, arrow) of the beat induced by the chirp.

In a behavioral study, Bastian et al. (2001) measured the probability of a fish emitting a small chirp (Fig. 7D). They observed a decrease in chirp probability with increasing beat frequency in a manner surprisingly similar to that reported here for the response gain of the electroreceptor afferents. Assuming that the fish emit chirps mainly at beat frequencies at which a conspecific fish would be able to detect the chirps, this match is consistent with our hypothesis that an enhanced firingfrequency response induced by a chirp is important for the detectability of a chirp. Although we are not able to make a causal connection between detectability of chirps in electroreceptor responses and behavior, and although it is not known from behavioral studies on which beat frequencies a fish is really able to detect a chirp, the single adaptation mechanism explaining our data could, in principle, account for a large part of the behavior. Moreover, this is consistent with the independence of the adaptation time constant from the electroreceptor $p$ value (baseline activity divided by EOD frequency), because this guarantees a well defined cutoff frequency to the higher-level neurons.

The important concept of a high-pass filter and its cutoff frequency relies on the linearity of the system under investigation. Because of their high baseline activity of $\sim 140-450 \mathrm{~Hz}$ (Bastian, 1981), their nicely linear $f-I$ curves around that baseline activity and the subtractive shift of the onset $f-I$ curve by adaptation $\mathrm{P}$-units are indeed well described by linear adaptation dynamics. Depressing synapses, on the other hand, exhibit similar signal transmission properties, in that they respond to changes in the input with strong transients (Abbott et al., 1997; Tsodyks and Markram, 1997; Tsodyks et al., 1998). However, depressing synapses are inherently nonlinear because of the fractional depletion of resources that differentiates them from the basically linear dynamics of spike-driven adaptation (Benda and Herz, 2003). Therefore, they might have more complex signal transmission properties.

The high-pass filter model introduced by Nelson et al. (1997) 
is equivalent to our model Equation 1 for the linear parts of the $f-I$ curves $\left(\tau_{\mathrm{a}}=\tau_{\mathrm{eff}}, G_{\mathrm{a}}=f_{0}^{\prime}-f_{\infty}^{\prime}, G_{\mathrm{b}}=0, G_{\mathrm{C}}=f_{\infty}^{\prime}\right)$. Longer adaptation time constants, as reported by Xu et al. (1996) and Nelson et al. (1997), do not play a role in encoding chirps, because the stimuli fluctuate symmetrically around the EOD baseline amplitude and usually contain frequencies $>1 \mathrm{~Hz}$. Furthermore, the additional threshold dynamics of an integrate-and-fire neuron introduced by Chacron et al. (2000) to explain negative ISI correlations (Hagiwara and Morita, 1963; Ratnam and Nelson, 2000) can be replaced by the adaptation dynamics of the high-pass filter that is added as an spike-activated adaptation current to a model for the spike generator. Thus, this single adaptation current of the electroreceptor afferents plays a threefold role in encoding behaviorally relevant stimuli. Because of negative ISI correlations, the spike-count variability to low-frequency stimuli is reduced, hence improving object detection (Ratnam and Nelson, 2000; Chacron et al., 2001). High-frequency stimuli including the transient induced by small chirps are transmitted with higher gain than low-frequency signals (Nelson et al., 1997; present study), and the negative ISI correlations additionally enhance the mutual information for high-frequency stimuli (Chacron et al., 2001).

Spike-driven adaptation and depressing synapses can produce many of the same input transformations. Both can account for negative ISI correlations in the output spike train (Goldman et al., 2002), and both respond to changes in the stimulus with strong transients. However, each depressing synapse filters its input individually, and the gain to each of the inputs of the neuron is therefore adjusted selectively (Abbott et al., 1997). In contrast, spike-driven adaptation acts on the overall input to the neuron and thus works like a master volume control. Spikedriven adaptation is observed in a wide range of neurons, and we propose that it may act to globally optimize detection of higherfrequency input. The combination of adaptation and synaptic depression may result in more complex computations. For example, avian wide-field tectal neurons (stratum griseum centrale cells) get input from retinal ganglion cells via depressing synapses. Together with an additional adaptation mechanism in the dendrites or soma, the cell performs spatiotemporal computations resulting in motion sensitivity independent of stimulus details (Luksch et al., 2004).

\section{Appendix}

\section{EOD AM as a separately processed stimulus}

The adaptation model Equation 1 was derived for currents directly stimulating the spike generator (Benda and Herz, 2003). However, in our study, we only used the AM as a stimulus, ignoring the fact that P-unit spikes are phase-locked to the EOD (Hagiwara et al., 1965) and that the spike generator therefore must be driven by the EOD. In other modeling studies of P-units, the EOD AM is processed independently from the EOD as well (Nelson et al., 1997; Chacron et al., 2000). Below, we show how the treatment of the EOD amplitude independent of the EOD carrier waveform could be justified.

The EOD waveform $s(t)$ can be approximated by a sine wave with angular frequency $\omega$, the amplitude of which is modulated by the stimulus $I(t)$ :

$$
s(t)=I(t) \sin (\omega t),
$$

[Note that in this notation, $I(t)$, includes the baseline EOD amplitude]. The EOD is picked up by $20-40$ receptor cells that synapse to a single nerve fiber (Zakon, 1987; Bennett et al., 1989). The transduction process and the transmission through the syn- apses might in its simplest case be described by a rectification of the stimulus, denoted by $[\cdot]_{+}$, which cuts off the negative parts of the argument, so that we get for the current $J(t)$ arriving at the spike generator:

$$
J(t)=b[s(t)]_{+},
$$

where $b=$ const is a proportionality factor. This current is converted into a series of spikes. Adopting the formalism of Benda and Herz (2003), the spike generation can be characterized by the firing frequency $f(t)$ given by the onset $f-J$ curve $\hat{f}_{0}(J)$ :

$$
f(t)=\hat{f}_{0}(\mathrm{~J}(\mathrm{t}))
$$

Assume that a weight function, $w(t)$, independent of $I(t)$, exists such that replacing the input current $J(t)$ in Equation 9 by its average

$$
\langle J\rangle_{\mathrm{T}, \mathrm{w}}(t):=\frac{1}{T} \int_{t-T / 2}^{t+T / 2} w\left(t^{\prime}-t\right) J\left(t^{\prime}\right) d t^{\prime}
$$

over a single ISI $T$ does not change the output firing frequency $f$ for any $I(t)$. Then we get the following:

$$
\langle J\rangle_{\mathrm{T}, \mathrm{w}}(t) \approx I(t) b\left\langle[\sin (\omega t)]_{+}\right\rangle_{\mathrm{T}, \mathrm{w}} .
$$

Because $T$ is usually greater than the period of the EOD, the average on the right can be approximated by a constant $c$. Absorbing $b$ and $c$ into the $f-I$ curve, we finally get the following:

$$
\hat{f}_{0}\left(\mathrm{~b}[\mathrm{I}(\mathrm{t}) \sin (\omega t)]_{+}\right) \approx \hat{f}_{0}(\mathrm{bcI}(\mathrm{t}))=\mathrm{f}_{0}(\mathrm{I}(\mathrm{t})),
$$

justifying the separated treatment of the AM $I(t)$ by the adaptation model Equation 1. Because our results obtained for AM stimuli are consistent with the model Equation 1, w(t) might likely exists. Thanks to the high baseline firing frequency of P-units of $\sim 140-450 \mathrm{~Hz}$, the cutoff frequency of the low-pass filter (10) is much higher than the one of the high-pass filter induced by adaptation, so that it does not interfere with our results. In summary, because of the integration (averaging) of the stimulus by the spike generator during ISIs that are usually longer than an EOD period, the treatment of the EOD AM independent of the EOD is possible.

\section{References}

Abbott LF, Varela JA, Sen K, Nelson SB (1997) Synaptic depression and cortical gain control. Science 275:221-224.

Baccus SA, Meister M (2002) Fast and slow contrast adaptation in retinal circuitry. Neuron 36:909-919.

Bastian J (1981) Electrolocation. I. How electroreceptors of Apteronotus albifrons code for moving objects and other electrical stimuli. J Comp Physiol [A] 144:465-479.

Bastian J, Schniederjan S, Nguyenkim J (2001) Arginine vasotocin modulates a sexually dimorphic communication behavior in the weakly electric fish Apteronotus leptorhynchus. J Exp Biol 204:1909-1923.

Benda J, Herz AVM (2003) A universal model for spike-frequency adaptation. Neural Comput 15:2523-2564.

Bennett MVL, Sandri C, Akert K (1989) Fine structure of the tuberous electroreceptor of the high-frequency electric fish, Sternarchus albifrons (gymnotiformes). J Neurocytol 18:265-283.

Castro-Alamancos MA (2004) Absence of rapid sensory adaptation in neocortex during information processing states. Neuron 41:455-464.

Chacron MJ, Longtin A, St-Hilaire M, Maler L (2000) Suprathreshold stochastic firing dynamics with memory in P-type electroreceptors. Phys Rev Lett 85:1576-1579.

Chacron MJ, Longtin A, Maler L (2001) Negative interspike interval correlations increase the neuronal capacity for encoding time-dependent stimuli. J Neurosci 21:5328-5343. 
Chung S, Li X, Nelson SB (2002) Short-term depression at thalamocortical synapses contributes to rapid adaptation of cortical sensory responses in vivo. Neuron 34:437-446.

Cook DL, Schwindt PC, Grande LA, Spain WJ (2003) Synaptic depression in the localization of sound. Nature 421:66-70.

Crook SM, Ermentrout GB, Bower JM (1998) Spike frequency adaptation affects the synchronization properties of networks of cortical oscillators. Neural Comput 10:837-854.

Engler G, Fogarty CM, Banks JR, Zupanc GKH (2000) Spontaneous modulations of the electric organ discharge in the weakly electric fish, Apteronotus leptorhynchus: a biophysical and behavioral analysis. J Comp Physiol [A] 186:645-660.

Ermentrout B, Pascal M, Gutkin B (2001) The effects of spike frequency adaptation and negative feedback on the synchronization of neural oscillators. Neural Comput 13:1285-1310.

Fairhall AL, Lewen GD, Bialek W, de Ruyter van Steveninck RR (2001) Efficiency and ambiguity in an adaptive neural code. Nature 412:787-792.

French AS, Höger U, Sekizawa SI, Torkkeli PH (2001) Frequency response functions and information capacities of paired spider mechanoreceptor neurons. Biol Cybern 85:293-300.

Fuhrmann G, Markram H, Tsodyks M (2002) Spike frequency adaptation and neocortical rhythms. J Neurophysiol 88:761-770.

Goldman MS, Maldonado P, Abbott LF (2002) Redundancy reduction and sustained firing with stochastic depressing synapses. J Neurosci 22:584-591.

Gollisch T, Herz AVM (2004) Input-driven components of spike-frequency adaptation can be unmasked in vivo. J Neurosci 24:7435-7444.

Hagedorn M, Heiligenberg W (1985) Court and spark: electric signals in the courtship and mating of gymnotoid fish. Anim Behav 33:254-265.

Hagiwara S, Morita H (1963) Coding mechanisms of electro-receptor fibers in some electric fish. J Neurophysiol 26:551-567.

Hagiwara S, Szabo T, Enger PS (1965) Electroreceptor mechanisms in a high-frequency weakly electric fish, Sternarchus albifrons. J Neurophysiol 28:784-799.

Kim KJ, Rieke F (2003) Slow $\mathrm{Na}^{+}$inactivation and variance adaptation in salamander retinal ganglion cells. J Neurosci 23:1506-1516.

Kohn A, Whitsel BL (2002) Sensory cortical dynamics. Behav Brain Res 20:119-126.

Luksch H, Khanbabaie R, Wessel R (2004) Synaptic dynamics mediate sen- sitivity to motion independent of stimulus details. Nat Neurosci 7:380-388.

Nelson ME, Xu Z, Payne JR (1997) Characterization and modeling of P-type electrosensory afferent responses to amplitude modulations in a wavetype electric fish. J Comp Physiol [A] 181:532-544.

Ratnam R, Nelson ME (2000) Nonrenewal statistics of electrosensory afferent spike trains: implications for the detection of weak sensory signals. J Neurosci 20:6672-6683.

Ronacher B, Hennig RM (2004) Neuronal adaptation improves the recognition of temporal patterns in a grasshopper. J Comp Physiol [A] 190:311-319.

Sanchez-Vives MV, Nowak LG, McCormick DA (2000) Membrane mechanisms underlying contrast adaptation in cat area 17 in vivo. J Neurosci 20:4267-4285

Senn W, Segev I, Tsodyks M (1998) Reading neuronal synchrony with depressing synapses. Neural Comput 10:815-819.

Sobel EC, Tank DW (1994) In vivo $\mathrm{Ca}^{2+}$ dynamics in a cricket auditory neuron: an example of chemical computation. Science 263:823-826.

Tsodyks M, Markram H (1997) The neural code between neocortical pyramidal neurons depends on neurotransmitter release probability. Proc Natl Acad Sci USA 94:719-723.

Tsodyks M, Pawelzik K, Markram H (1998) Neural networks with dynamic synapses. Neural Comput 10:821-835.

van Vreeswijk C, Hansel D (2001) Patterns of synchrony in neural networks with spike adaptation. Neural Comput 13:959-992.

Wang XJ (1998) Calcium coding and adaptive temporal computation in cortical pyramidal neurons. J Neurophysiol 79:1549-1566.

Wang XJ, Liu Y, Sanchez-Vives M, McCormick D (2003) Adaptation and temporal decorrelation by single neurons in the primary visual cortex. J Neurophysiol 89:3279-3293.

Xu Z, Payne JR, Nelson ME (1996) Logarithmic time course of sensory adaptation in electrosensory afferent nerve fibers in a weakly electric fish. J Neurophysiol 76:2020-2032.

Zakon HH (1987) Variation in the mode of receptor cell addition in the electrosensory system of gymnotiform fish. J Comp Neurol 262:195-214.

Zupanc GKH, Maler L (1993) Evoked chirping in the weakly electric fish Apteronotus leptorhynchus: a quantitative biophysical analysis. Can J Zool 71:2301-2310. 